TI 2011-096/3

Tinbergen Institute Discussion Paper

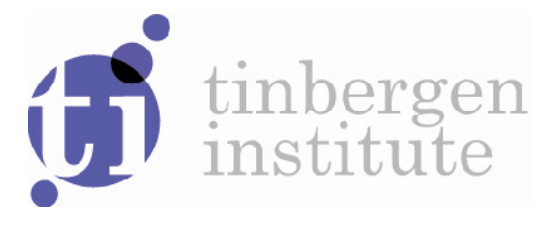

\title{
Teleworking and Congestion: A Dynamic Bottleneck Analysis
}

Sergejs Gubins

Erik T. Verhoef

Dept. of Spatial Economics, Faculty of Economics and Business Administration, VU University of Amsterdam, and Tinbergen Institute. 
Tinbergen Institute is the graduate school and research institute in economics of Erasmus University Rotterdam, the University of Amsterdam and VU University Amsterdam.

More TI discussion papers can be downloaded at http://www.tinbergen.nl

Tinbergen Institute has two locations:

Tinbergen Institute Amsterdam

Gustav Mahlerplein 117

1082 MS Amsterdam

The Netherlands

Tel.: +31(0)205251600

Tinbergen Institute Rotterdam

Burg. Oudlaan 50

3062 PA Rotterdam

The Netherlands

Tel.: +31(0)10 4088900

Fax: +31(0)104089031

Duisenberg school of finance is a collaboration of the Dutch financial sector and universities, with the ambition to support innovative research and offer top quality academic education in core areas of finance.

DSF research papers can be downloaded at: http://www.dsf.nl/

Duisenberg school of finance

Gustav Mahlerplein 117

1082 MS Amsterdam

The Netherlands

Tel.: +31(0)20 5258579 


\title{
Teleworking and Congestion: A Dynamic Bottleneck Analysis
}

\author{
Sergejs Gubins", Erik T. Verhoef \\ Department of Spatial Economics, VU University Amsterdam, De Boelelaan 1105, 1081 HV Amsterdam, \\ The Netherlands \\ Tinbergen Institute, Gustav Mahlerplein 117, 1082 MS Amsterdam, The Netherlands
}

\begin{abstract}
We analyze the welfare effects of part-day teleworking on road traffic congestion in the context of Vickrey's dynamic bottleneck model. Endogenous decisions to become equipped with a teleworkingenabling technology change the commuting pattern of equipped drivers and, due to congestion externalities, affect travel costs of all drivers. We show that even costless teleworking might be marginally welfare reducing, after reaching the optimal penetration level, as an equipped driver imposes a higher travel externality on other equipped drivers than unequipped drivers do. We find that private monopolistic supply of the technology might yield a higher social welfare than perfectly competitive supply.
\end{abstract}

JEL classification: $\quad$ D62, O33, R41, R48

Keywords: $\quad$ teleworking, congestion, bottleneck

\footnotetext{
${ }^{*}$ Corresponding author. Tel.: +31 205988106.

E-mail addresses: s.gubins@vu.nl (S. Gubins), e.t.verhoef@vu.nl (E.T. Verhoef).

Financial support from The Netherlands Organization for Scientific Research (NWO) is gratefully acknowledged. This paper is part of TRISTAM project (Traveler Response and Information Service Technology - Analysis and Modeling). Also the financial support from the ERC (Advanced Grant OPTION \#246969) for the research of Erik Verhoef is gratefully acknowledged. We thank for useful discussions and comments Thomas de Graaff, seminar participants at Tinbergen Institute and BICEPS (Riga, Latvia), and conference participants at BIVEC-GIBET (Namur, Belgium), Kuhmo-Nectar (Stockholm, Sweden) and NARSC (Miami, USA).
} 


\section{Introduction}

Road congestion is a challenging and persisting problem. Various policy measures have been proposed to tackle congestion, including investment in transport infrastructure and public transit, provision of traffic information to drivers, city zoning, road pricing, parking policies and flexible working hours. Since Pigou (1920), most economists agree that marginal cost pricing of roads offers the first-best solution to congestion problems; see, for example, the exposition in Small and Verhoef (2007). However, optimal tolling seems technically hard to implement in practice; and in part due to its redistributive effect, pricing suffers from low political acceptability that further hinders a wide implementation. There are only a few cities, the best-known being Singapore, Stockholm, and London, with road pricing schemes, usually in the form of a fixed or step cordon toll. The limited feasibility of the first-best policy motivates an ongoing search for alternatives.

Teleworking is one such possible alternative. It refers to out-of-office work arrangements, usually from home and sometimes with flexible time schedules. Whole-day teleworking allows an individual to avoid commuting between the home and the workplace altogether, while part-day teleworking could make it easier to circumvent congestion by commuting during off-peak hours. Progress in information and communication technologies (ICT), such as the availability of the remote access to secured databases, cloud computing, networks and a general advance of Internet technologies, expands both the intensive and the extensive margin of teleworking use. Moreover, governments stimulate teleworking use. For instance, in the USA, the Telework Enhancement Act of 2010 promotes teleworking among public servants. Given the range of potential benefits on labor productivity, work-life balance, job matching, and given expected future technological progress, one may expect teleworking to be of increasing relevance in the future. ${ }^{1}$

Against this background, this paper will investigate the effects of part-day (morning) teleworking on congestion, from the economic perspective. Part-day teleworking is an empirically relevant phenomenon; for example, one UK survey shows that in 2007 part-day teleworking had a higher incidence rate among full-time employees than whole-day teleworking, with diffusion rates of 17.2 and 9.8 respectively (Haddad, Lyons and Chatterjee, 2009). In the context of this paper, one might think of the employees performing some work tasks from home in the morning, and then coming to an office for the rest of the workday.

Studies that do model the impacts of teleworking on travel typically focus on the spatial dimension, notably to capture the long-term effects of whole-day teleworking on residential choice within a city; see, among others, Safirova (2002), Rhee (2008) and a short subsection on job decentralization in Glaeser (2008, p. 41). But time-of-day adjustments may also be relevant, especially for part-day teleworking. To effectively incorporate this temporal aspect of part-day teleworking, we apply Vickrey's

\footnotetext{
${ }^{1}$ Increase of the teleworking incidence rate over time has occurred in the past. According to the Eurofound surveys (2005, 2010), an employees' self-reported EU average incidence rates of teleworking for at least one-quarter of their time were 4 and 7 percents in, respectively, 2000 and 2005, with large variation across countries, industries and days of the week.
} 
(1969) dynamic bottleneck model, a workhorse model in transportation economics, in which the drivers' scheduling decisions are endogenous.

We model the behavioral impacts of teleworking by assuming that access to the teleworking-enabling ICT raises the utility that an individual derives from being at home at any given point in time. We therefore define a teleworking individual as a person who is equipped with a technology that allows her to perform various work tasks from home. An equipped individual values time spent at home higher than an unequipped one, and, as we show later, has an incentive to therefore postpone the arrival time at work. The choice of whether to be equipped is determined within the model; thus our model may produce endogenous heterogeneity of drivers when not everybody chooses to become equipped. We derive an inverse demand for the teleworking technology, and show that the marginal willingness to pay depends negatively on the number of teleworking people, due to the relatively large congestion externality equipped drivers impose on one another. We show that even costless technology might be marginally welfare reducing after teleworking reaches a certain optimal penetration level. We also study private market provision of the teleworking technology, both under perfect competition and monopoly, and define conditions when the social welfare is found to be higher under monopoly.

Our study fits in a wider literature that considers the potential impacts of ICT on congestion and social welfare. However, most of this literature focuses on the provision of traffic information to drivers; see for example, Arnott, de Palma and Lindsey (1996); de Palma and Lindsey (1998); and Emmerink, Verhoef, Nijkamp and Rietveld (1998a, 1998b). These studies consistently show that under an unpriced congestion externality, the marginal effect of information might sometimes be welfare decreasing. To the best of our knowledge, our study is the first to show this in the context of teleworking. Given the popularity of ICT-based solutions to traffic congestion, these results are important for practical policy making.

The paper is organized as follows. Section 2 introduces Vickrey's dynamic bottleneck model, and teleworking within that framework. Section 3 derives the marginal willingness to pay for, and social benefits of teleworking. Section 4 considers private provision of the teleworking technology in markets of perfect competition and monopoly. We evaluate the relative efficiency of market outcomes, compared to the social optimum. Section 5 considers the impact of teleworking on travel in case firstbest road tolling already addresses the congestion externality. Section 6 summarizes the paper, highlights the main findings, and concludes with a list of possible extensions.

\section{Teleworking within Vickrey's dynamic bottleneck model}

\subsection{Basic model}

Our analysis is cast in the framework of Vickrey's (1969) dynamic bottleneck model, which provides a stylized description of traffic congestion at a single traffic bottleneck. The model builds upon the observation that traffic congestion in reality is a dynamic phenomenon; with waiting times and queues 
first increasing over time during the rush hour, and subsequently declining. Vickrey's model explicitly considers the decisions of drivers to start travel at certain moments in time, and applies a dynamic equilibrium condition in which no driver can be better off by unilaterally changing the departure time.

In its simplest form the dynamic bottleneck model considers a morning period during which $\bar{N}$ homogeneous atomistic car drivers decide on the time of departure from a single origin ("home") to a single destination ("workplace"). Drivers have the same preferred arrival time at work, $t^{*}$, a deviation from which causes a driver to incur a schedule delay cost. There is a road bottleneck in between home and work, possibly a bridge or a tunnel, with a capacity $s$. Thus, a "first-in first-out" traffic jam starts to build up after the flow of drivers arriving to the bottleneck has first exceeded its capacity. Each minute of spending time in a traffic jam results in a travel delay cost for a driver. The free-flow travel time is set to zero, without loss of generality in this context, implying that without a queue, drivers depart from home, pass the bottleneck and arrive at work all at the same moment.

The dynamic bottleneck model highlights an important equilibrating mechanism affecting behavior in a traffic jam: the trade-off that drivers make between schedule delay costs of arriving at an inconvenient time, versus the travel delay cost of waiting in the queue. This stylized description of traffic congestion offers a framework for studying dynamic departure time decisions, and the dynamic evolution of traffic conditions over the rush hour period, within one analytical model that lends itself to closed-form solutions of optima and equilibria (e.g., Arnott, de Palma and Lindsey, 1993).

The standard dynamic bottleneck model uses a linear cost function, in which there is a time-invariant value of travel time $(\alpha)$, a constant unit shadow price of schedule delay for arriving early $(\beta)$, and one of schedule delay for arriving late $(\gamma)$. This scheduling setup is attributed to Vickrey (1969) and Small (1982), and now extensively used in the transportation economics literature. Vickrey (1973), and later Tseng and Verhoef (2008), considered a somewhat more general specification of scheduling behavior and utility, which explicitly describes the underlying pattern of activities in terms of clock-timedependent utilities of being at various locations. This approach is useful for our purposes, as it allows us to incorporate the impact of teleworking technology in the bottleneck model in a structured way. In this approach, a driver derives utility from being either at home $(H(t)$ per unit of time), at work (W(t) per unit of time), or in a vehicle $\left(V(t)\right.$, standardized to zero for convenience). ${ }^{2}$ While many functional utility specifications for $H(t)$ and $W(t)$ are possible, Tseng and Verhoef (2008) show that only one particular specification is equivalent to the standard linear (" $\alpha-\beta-\gamma$ ") scheduling setup. In this specification, $H(t)$ is equal to some constant ( $H$ henceforth) throughout the period considered, while $W(t)$ is piecewise constant with an upward jump at $t^{*}$, and $W(t)=W_{E}<H$ before $t^{*}$ and $W(t)=W_{L}>H$ after $t^{*}$. The equivalence with the conventional linear function stems from the fact that the opportunity cost of being at work before $t^{*}$ is, then, $H-W_{E}=\beta$; the opportunity cost of not being at work after $t^{*}$ is $W_{L}-H=$ $\gamma$; and the opportunity cost of being in a vehicle is $H-V=\alpha$. The usual assumption that $\alpha>\beta$ translates into $V(t)=0<W(t)$, and reflects that a driver prefers to enter the workplace above staying in the car after having passed the bottleneck. We plot this utility structure in Figure 1.

\footnotetext{
${ }^{2}$ Strictly speaking, $H, W$ and $V$ are Marshallian surpluses, which are the ratios of marginal utility of time spend at respectively, home, work and in a vehicle, over marginal utility of income. For brevity we refer to them as utilities.
} 
Figure 1. Utility structure and opportunity costs for a driver in the conventional representation of Vickrey's dynamic bottleneck model

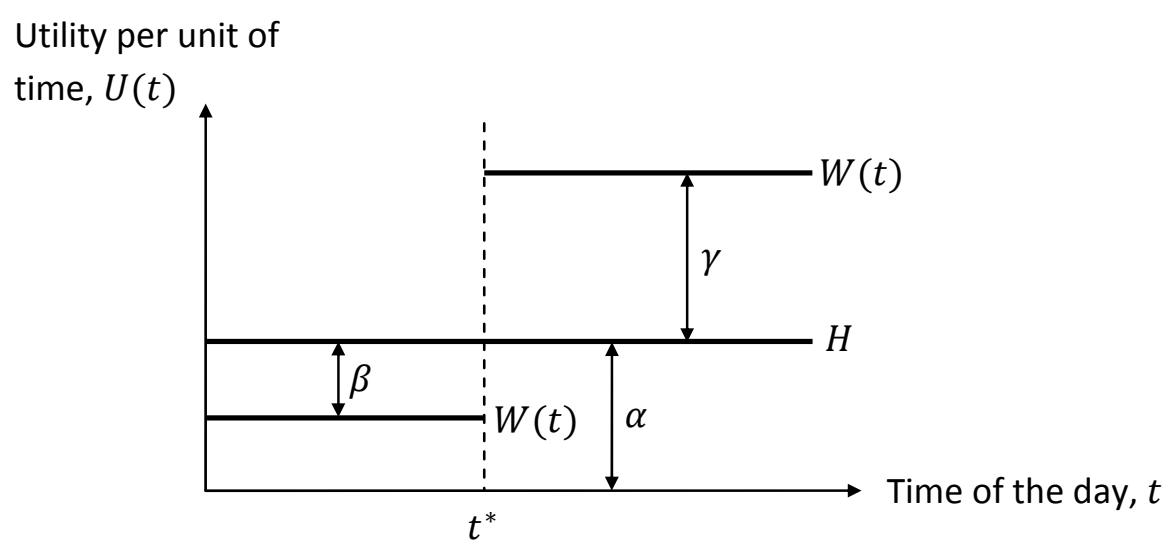

Based on Tseng and Verhoef (2008), Figure 2.

We use the linear utility specification primarily to stay as close as possible to the conventional linear scheduling setup which assumes constancy of $\alpha, \beta$ and $\gamma$; and which has been applied in most of the bottleneck model literature. Furthermore, we see no immediate reasons why the model's insights on the desirability of different market structures for the supply of teleworking technology would depend critically on this specific functional form.

With inelastic demand, the single margin of behavior in the bottleneck model is the arrival time at work, $t$, which a driver sets to maximize utility over the course of the morning. We define the morning period such that it starts for every driver at the common time $t_{S}$ and finishes at $t_{F}$ (chosen such that the interval is wide enough to cover the entire congestion period, or peak). An individual's utility level is then equal to an "ideal" utility level $\Gamma$, which she would reach over the course of the morning, had she both departed and arrived at the preferred arrival time $t^{*}$, minus the generalized travel cost that she actually incurs. The latter consists of travel delay and schedule delay costs, and will be denoted $P(t)$. A driver incurs schedule delay cost when the time of her arrival at work is not $t^{*}$. Each minute of arriving at work either early or late has a value of, respectively, $\beta$ and $\gamma$. In turn, each minute of travel delay, $T(t)$, has a value of time $\alpha$. The reader can verify this in Figure 1 by evaluating the costs for the three types of trips that are possible when they can start and end before or after $t^{*}$.

Thus, the utility level of a driver arriving at work at time $t$ is:

$$
U(t)=\Gamma-P(t)=\alpha\left(t^{*}-t_{S}\right)+(\alpha+\gamma)\left(t_{F}-t^{*}\right)-\alpha T(t)- \begin{cases}\beta\left(t^{*}-t\right) & \mid t \leq t^{*} \\ \gamma\left(t-t^{*}\right) & \mid t>t^{*}\end{cases}
$$


Note that the first two terms are constants, and the final two (time-depended) terms correspond to the conventional generalized cost of travel.

To determine the dynamic equilibrium (see also Vickrey, 1969; and Arnott, de Palma and Lindsey, 1993), first observe that in a dynamic equilibrium, the utility levels of all (homogeneous) drivers must be equal; i.e., no one is able to unilaterally adjust her arrival time and consequently gain in utility. The very first driver arrives at work at time $t_{q}$ and faces no travel delay costs, as she freely passes the bottleneck. But she incurs schedule delay costs from being early at work; thus, her generalized cost is $\beta\left(t^{*}-t_{q}\right)$. Likewise, the very last driver who arrives at time $t_{q}$, incurs the cost of arriving late at work but again faces no travel delay; the generalized travel cost is then $\gamma\left(t_{q},-t^{*}\right)$. Because the ideal utility level $\Gamma$ is identical across homogeneous drivers, the equilibrium condition implies equality of generalized travel costs. Given that the duration of the peak period is $\frac{\bar{N}}{s}=\left(t_{q}-t_{q^{\prime}}\right)$, the peak period starts and ends at:

(2) $t_{q}=t^{*}-\frac{\gamma}{\beta+\gamma} \frac{\bar{N}}{s}$
(3) $t_{q^{\prime}}=t^{*}+\frac{\beta}{\beta+\gamma} \frac{\bar{N}}{s}$

Each driver therefore faces generalized travel costs equal to:

(4) $\quad P(t)=\frac{\beta \gamma}{\beta+\gamma} \frac{\bar{N}}{s}$

The driver who arrives at work at time $t^{*}$ only incurs travel delay cost $\alpha T\left(t^{*}\right)$. In equilibrium, she has the same generalized travel cost as the first driver; hence her waiting time in a traffic jam is $T\left(t^{*}\right)=\frac{\beta \gamma}{\beta+\gamma} \frac{\bar{N}}{s} \frac{1}{\alpha}$.

In equilibrium, when generalized costs are identical across drivers, for those who arrive before $t^{*}$ each additional one minute arrived closer to $t^{*}$ decreases the schedule delay cost by $\beta$, but must increase travel delay by $\frac{\beta}{\alpha}$ to keep generalized cost constant over time. In the same fashion, for arrivals later than $t^{*}$ the travel delay decreases with $\frac{\gamma}{\alpha}$ by arrival time to keep generalized cost constant. Figure 2 shows the equilibrium combinations of arrival times and travel delays. The slopes of the triangle naturally depends on the parameters $\alpha, \beta, \gamma$; while the width depends on $\frac{\bar{N}}{s}$, which determines the duration of the peak (the time interval between $t_{q}$ and $t_{q}$ ). As the generalized costs are constant over time, one may interpret the graph as an isocost function. As there are no arrival times with a generalized cost level below the equilibrium level, and given that vehicles are treated as a continuum, the equilibrium in Vickrey's dynamic bottleneck model is a Nash equilibrium. 
Figure 2. Equilibrium isocost function with homogeneous drivers

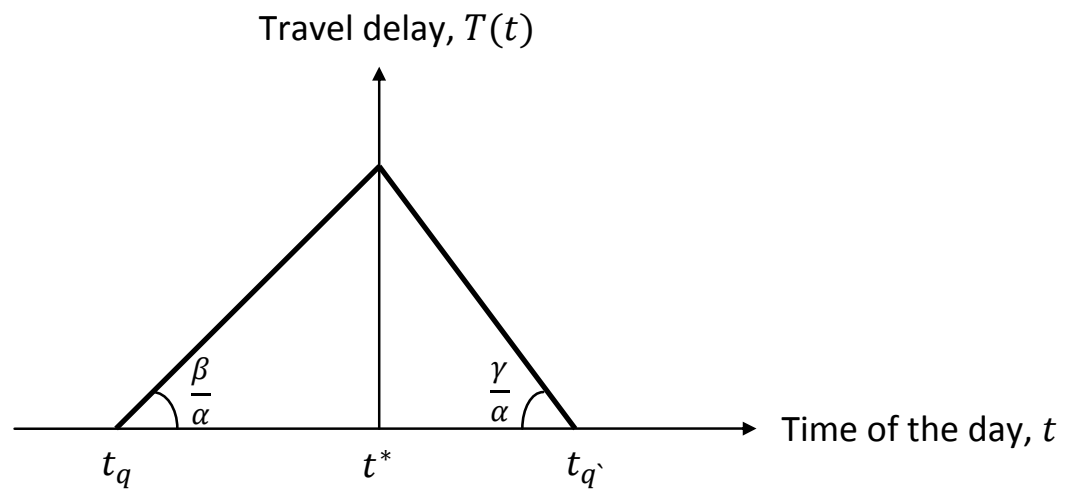

\subsection{Introduction of teleworking technology}

The framework shown in Figure 1 helps us to make a structured and well-motivated assumption on how the availability of teleworking technology would affect the value-of-time components $\alpha, \beta$ and $\gamma$. Maintaining a piecewise constant utility structure, and assuming that the technology would affect the utility of being at home (not of being at work or in the vehicle), the natural assumption to make is that it shifts $H$ upwards; and does so by some constant as we want to maintain the qualitative pattern displayed in Figure 1. This means that, in terms of the conventional scheduling formulation, adaptation of teleworking technology will lead to equally large increases in $\alpha$ and $\beta$, and a decrease in $\gamma$ that is equally large in absolute size. Intuitively, an individual equipped with the teleworking technology would put a higher value on time spent at home before $t^{*}$, as being at home results in higher utility due to the possibility of teleworking. At the same time, for an equipped driver, who arrives at work after $t^{*}$, a trip does not cause as much cost as for an unequipped one, as the teleworker may reduce the disutility of a late arrival at work by working from home. And the conventional value of travel time $\alpha$ increases, because the opportunity cost of not being at home increases.

A constant shift in $H$ due to the teleworking technology implies that an equipped driver starts gaining higher utility from being at home right after the beginning of the morning, at $t_{S}$. That might represent that an equipped driver works during the entire morning, or, alternatively, that due to the availability of technology, an individual is able to reschedule other activities (not modeled explicitly) at home in such a way that the utility (equalized over time) derived from being at home rises.

Of course, other assumptions could have been made on how teleworking would affect the utility function. We believe our assumption captures the most relevant aspect of the issue, in the simplest possible utility specification. Specifically, only with a constant upward shift of $H$ do we have a utility function that can be characterized by the three conventional constant shadow prices $\alpha, \beta$ and $\gamma$ both before and after an individual is equipped.

We thus assume that the technology raises the unit value of staying at home by a constant $\Delta$, for which we assume $\Delta<\gamma$. The latter inequality assures that also those drivers who are equipped with the 
teleworking technology still find it worthwhile to be at work at times $t \geq t^{*}$. Note from Figure 1 that our specification leaves the preferred arrival time $t^{*}$ unchanged. This is in fact a welcome feature, because it secures that any predicted shift of the peak period that results from adaptation of technology by drivers, which we will indeed find in our model, can be ascribed solely to the impact of changes in $H$, $\alpha, \beta$ and $\gamma$; and not to a change of $t^{*}$.

\section{Marginal willingness to pay for and externalities of the teleworking technology}

\subsection{Marginal willingness to pay for teleworking technology}

In this section we derive the marginal willingness to pay for acquiring the teleworking technology (MWTP). We will show that this willingness to pay depends on the aggregate level of technology penetration: if more drivers are equipped with the technology, an individual driver is willing to pay less for it. The intuition is that the equipped drivers tend to postpone their departure times to be "at the end of the peak" to gain most benefit from teleworking, but the rising number of the equipped drivers makes the end of the peak increasingly late, and this diminishes teleworking benefits and therewith marginal willingness to pay for it. Later we capture this result in Proposition 1, after first having established Lemmas 1 and 2 below.

The marginal willingness to pay for the teleworking technology is the difference between the utility that a driver reaches over the course of the morning when being equipped with the technology, $U_{e}$, minus the utility when being unequipped, $U_{u}$. As follows from Equation (1), changes in opportunity $\operatorname{costs} \alpha, \beta$ and $\gamma$, affect an individual's utility $U_{e, u}$ via a change in the ideal utility, $\Gamma_{e, u}$, and in the generalized travel costs that one incurs, $P_{e, u}$, where subscripts $e$ and $u$ denote, respectively, equipped and unequipped drivers:

$$
M W T P=U_{e}-U_{u}=\left(\Gamma_{e}-P_{e}(t)\right)-\left(\Gamma_{u}-P_{u}(t)\right)
$$

The derivation of the ideal utility values $\Gamma_{e}$ and $\Gamma_{u}$ is straightforward. A driver equipped with teleworking technology has a higher ideal utility than an unequipped driver, because between $t_{S}$ and $t^{*}$, a higher utility of being at home is enjoyed. ${ }^{3}$ This increase in ideal utility is, of course, identical for all teleworkers, and does not depend on one's arrival time at work.

The derivation of equilibrium cost values $P_{e}(t)$ and $P_{u}(t)$ is more involved. Both equipped and unequipped drivers choose their arrival time at work $t$ to minimize generalized travel costs. With different opportunity costs, the slopes of isocost functions as shown in Figure 2 may differ between

\footnotetext{
${ }^{3}$ The difference in ideal utility levels of equipped drivers $\Gamma_{e}$ and unequipped ones $\Gamma_{u}$ is $\Gamma_{e}-\Gamma_{u}=\Delta\left(t^{*}-t_{S}\right)>0$ whenever $t^{*}>t_{S}$ and $\Delta>0$, as we assume.
} 
drivers. Therefore, a driver who adopts the technology may have an incentive to change the arrival time at work, in order to minimize generalized travel costs under the new time values.

Dynamic equilibrium requires that for both groups of travelers, if both are greater than zero in size, the generalized travel costs are equal at moments when arrivals occur, and not lower at other times. We will see shortly that this will involve temporal separation of travelers when both types exist.

To see why this occurs, first note that the upper envelope of the groups' equilibrium isocost functions corresponds to the equilibrium pattern of travel delays. Let $N_{e}$ be the number of equipped drivers, and $N_{u}^{L}$ and $N_{u}^{E}$ the numbers of unequipped drivers who arrive at work, respectively, after ("Late") and before ("Early") $t^{*}$. For a fixed overall number of drivers, $\bar{N}$, the duration of the peak period will be $\frac{\bar{N}}{s}=\frac{N_{e}}{s}+\frac{N_{u}^{L}}{s}+\frac{N_{u}^{E}}{s}$. The timing of the beginning of the peak, however, is endogenous.

To determine the equilibrium level of generalized cost, we have to distinguish between two cases, one with relatively low numbers of equipped drivers $\left(0 \leq N_{e} \leq N_{e}^{\#}\right)$, and another with relatively high numbers $\left(N_{e}^{\#}<N_{e} \leq \bar{N}\right)$, where $N_{e}^{\#}$ will be defined later. With low numbers, equipped drivers will arrive only after $t^{*}$. Figure 3 illustrates this type of equilibrium, and shows the equilibrium isocost functions for both types of drivers. The isocost lines of unequipped drivers have slopes $\frac{\beta}{\alpha}$ and $-\frac{\gamma}{\alpha}$; those of equipped drivers have slopes $\frac{\beta+\Delta}{\alpha+\Delta}$ and $-\frac{\gamma-\Delta}{\alpha+\Delta}$. The slopes for equipped drivers are, therefore, steeper for early arrivals (recall that $\beta<\alpha$ ), and flatter for late ones.

Figure 3. Isocost functions of the heterogeneous drivers, if equipped drivers arrive at work late

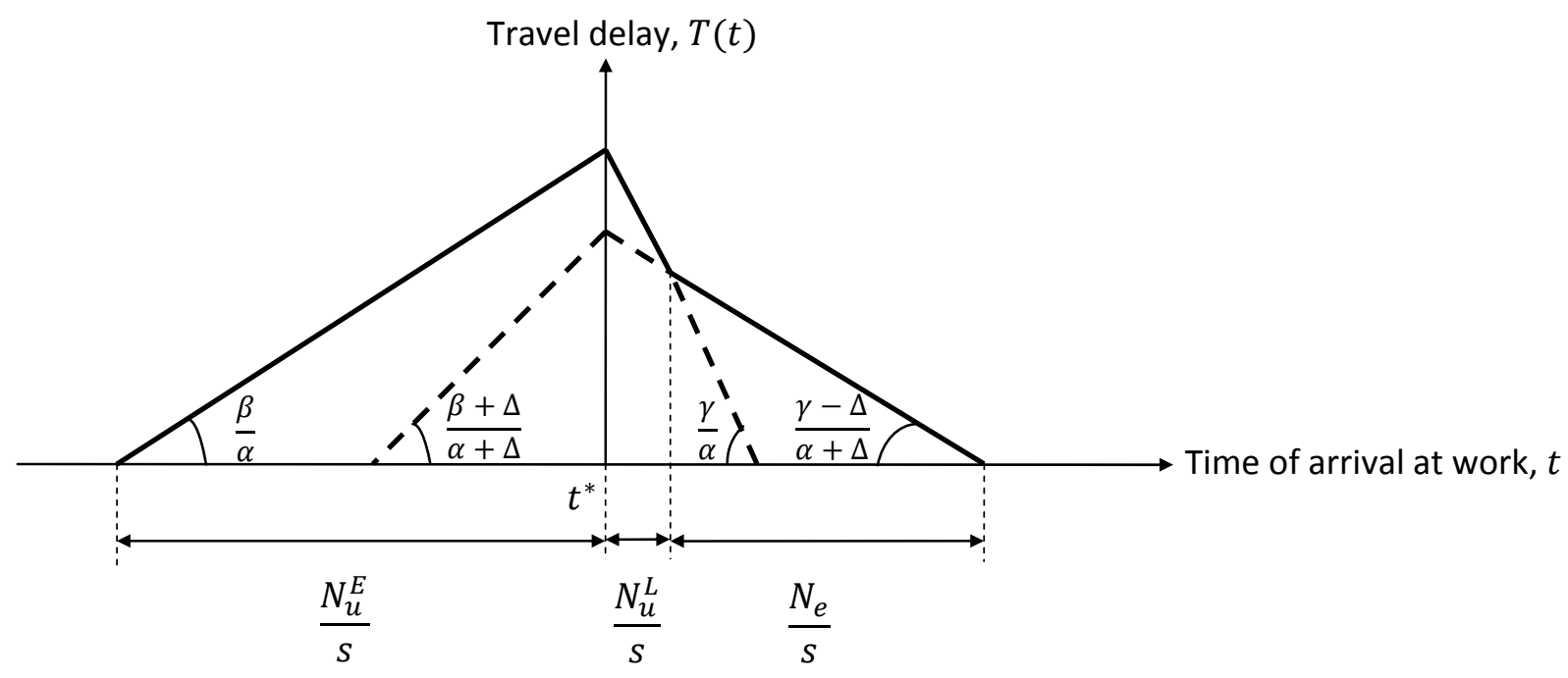

It is very easy to prove that the first single (atomistic) driver who gets equipped prefers to be the last traveler to pass the bottleneck. Given the equilibrium isocost line for unequipped drivers, which gives the equilibrium pattern of travel times for this group, the last arrival time brings the single equipped 
driver at the lowest achievable isocost line. This driver spends the peak period at home, benefits from teleworking, and then travels to work, incurring generalized travel costs of $\frac{\beta(\gamma-\Delta)}{\beta+\gamma} \frac{\bar{N}}{s}$. As more drivers become equipped, their equilibrium isocost function will shift upwards to accommodate the increasing number $N_{e}$. At the same time, the equilibrium isocost of the unequipped group moves downward, as there is decreasing demand for early arrivals.

The different slopes of isocost lines in Figure 3 thus induce a temporal separation of travelers, where equipped drivers arrive later than unequipped ones. We summarize this result in Lemmas 1 and 2.

Lemma 1. The generalized travel costs for unequipped drivers $P_{u}$ is decreasing when the share of equipped drivers $N_{e}$ rises, i.e., $\frac{\partial P_{u}}{\partial N_{e}}<0$.

Proof. See Appendix.

The proof is straightforward; it entails deriving the duration of the interval where unequipped drivers arrive after $t^{*}\left(\frac{N_{u}^{L}}{s}\right)$ and the duration of the peak before $t^{*}\left(\frac{N_{u}^{E}}{s}\right)$. The former is non-negative if $N_{e}$ is below the level that defines the threshold value $N_{e}^{\#}$ :

$$
N_{e}^{\#}=\frac{\beta(\alpha+\Delta)}{\beta(\alpha+\Delta)+\alpha(\gamma-\Delta)} \bar{N}
$$

Lemma 1 implies that equipped drivers in some sense impose a positive external effect on unequipped drivers. More precisely, they impose a smaller external cost on unequipped drivers than unequipped drivers do themselves. The underlying reason is that the groups have different preferences for arrival time adjustments, where equipped drivers have a less strong demand for early arrivals.

Lemma 2. The generalized travel costs for equipped drivers $P_{e}$ is increasing when the share of equipped drivers $N_{e}$ rises, i.e., $\frac{\partial P_{e}}{\partial N_{e}}>0$.

Proof. See Appendix.

Lemma 2 implies that equipped drivers impose a negative marginal externality on their own group, that exceeds the negative externality that unequipped drivers impose on equipped drivers. 
Using results on generalized cost levels derived for Lemmas 1 and 2, we can find an expression for equation (5) which immediately leads to Proposition 1.

Proposition 1. MWTP is negatively related to the number of equipped drivers $N_{e}$, so if more drivers are equipped, an additional individual driver is willing to pay less for teleworking technology, i.e., $\frac{\partial M W T P}{\partial N_{e}}<0$.

Proof. See Appendix.

That $M W T P$ decreases with the rise of $N_{e}$ is true for both the marginal unequipped driver who becomes equipped, and also for the already equipped drivers, as these will have the same benefits of remaining equipped as the marginal equipped driver. Hence, the total benefits for the equipped drivers collectively amounts to $M W T P \cdot N_{e}$.

The slope of $M W T P$ in the range $N_{e}^{\#}<N_{e} \leq \bar{N}$, is flatter than in the range $0 \leq N_{e} \leq N_{e}^{\#}$, resulting in a kink in the MWTP function at $N_{e}^{\#}$. The reason for the kink is the difference in externalities imposed by early versus late equipped drivers. Figure 4 illustrates the $M W T P$ as a function of $N_{e}$ (see derivations in the proof of Proposition 1 in Appendix). Although the MWTP is declining, also the very last driver to become equipped has a positive willingness to pay, as we set the start of the day $t_{S}$ before the first driver arrives at work even when teleworking possibility is not available (i.e., $t^{*}-t_{S} \geq \frac{\gamma}{\beta+\gamma} \frac{\bar{N}}{s}$ ). Under this constraint, the MWTP value at $\bar{N}$, as shown in Figure 4, is positive. A positive MWTP also for the last driver to become equipped is consistent with the notion that even when not changing departure time, this driver has benefited at a rate $\Delta$ over the time spent at home between the start of the day and the moment of departing.

Figure 4. Marginal willingness to pay for teleworking technology

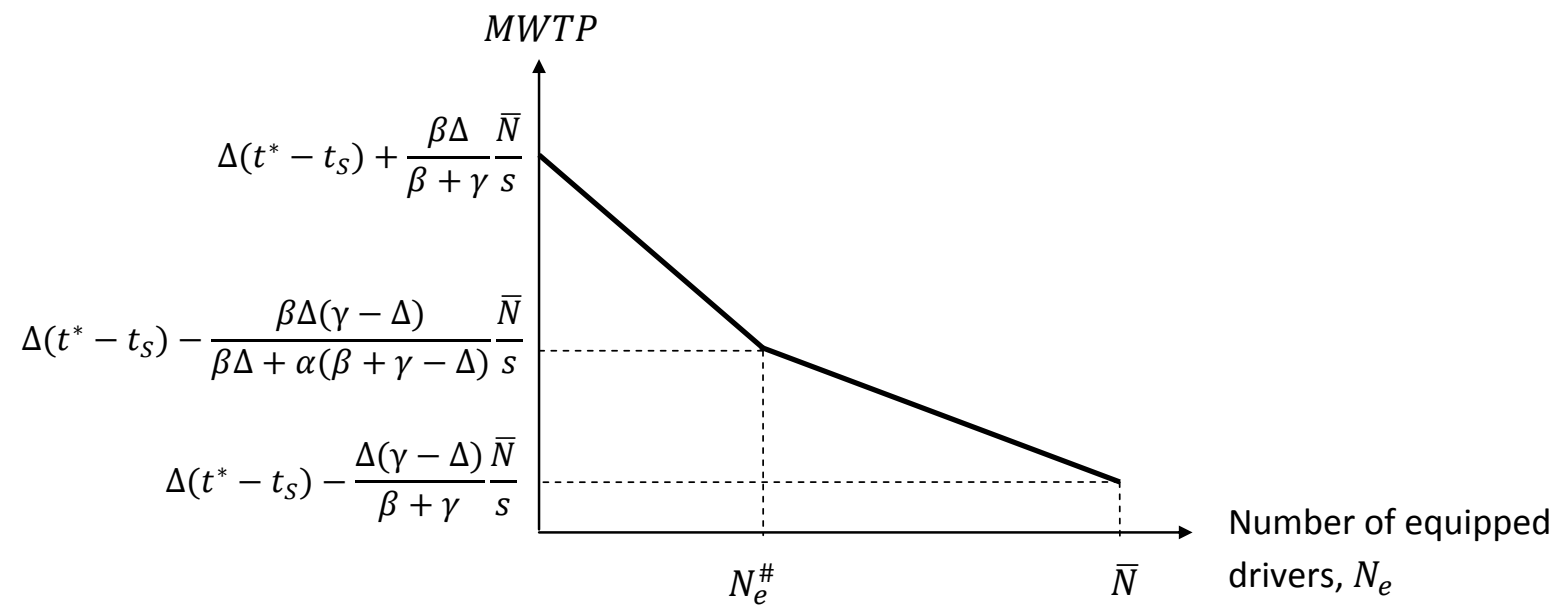




\subsection{Marginal social benefit function}

All drivers' generalized travel costs change when an additional unequipped driver becomes equipped. More precisely, with each additional driver becoming equipped, the already equipped drivers will incur higher costs, while unequipped drivers' costs decrease. Changes in costs for other drivers are external to the driver who becomes equipped. The (private) marginal willingness to pay function therefore does not correspond to the marginal social benefit function, as the latter encompasses these externalities.

To calculate the socially optimal level of teleworking, we derive the marginal social benefit function of the teleworking technology $(M S B)$. With zero marginal cost, the adaptation of the technology by an additional driver is socially desirable as long as $M S B$ is non-negative; otherwise the number of teleworkers is socially excessive.

To determine the $M S B$, we distinguish between three types of drivers: the single (atomistic) driver who becomes equipped, the drivers already equipped $\left(N_{e}\right)$, and those unequipped $\left(\bar{N}-N_{e}\right)$. MSB is then equal to the sum of marginal willingness to pay $(M W T P)$ of the driver who is becoming equipped, minus the marginal external costs for all equipped drivers, plus the marginal external benefits ${ }^{4}$ to all unequipped drivers. The marginal external benefits to all unequipped drivers is the derivative of their generalized travel costs with respect to number of equipped drivers (recall that the total number of drivers is fixed, so the reduction in the number of unequipped drivers is also accounted for), multiplied by the number of unequipped drivers:

(7) $\quad M S B_{u}=-\frac{\partial P_{u}}{\partial N_{e}}\left(\bar{N}-N_{e}\right)$

And similarly for equipped drivers:

(8) $\quad M S B_{e}=-\frac{\partial P_{e}}{\partial N_{e}} N_{e}$

One can also determine the $M S B$ by taking the derivative of the total generalized travel costs of all drivers jointly with respect to the number of equipped drivers. $M S B$ is then minus the resulting derivative, plus the increase in ideal utility for a single driver who is getting equipped. It has been verified that the two approaches lead to the same result.

\footnotetext{
${ }^{4}$ For brevity we refer to the decrease in marginal external cost for the unequipped driver as if it was a marginal external benefit.
} 
The marginal social benefit function is then the sum of $M W T P, M S B_{u}$ and $M S B_{e}$. We define $M S B$ separately for both relevant ranges, of "low" and "high" number of equipped drivers. The slopes and intercepts of $M S B$ over those two ranges differ, and overall $M S B$ is discontinuous at $N_{e}^{\#}$. This discontinuity stems from the differences in external effects that drivers impose upon one another in early arrivals compared to late ones. Comparing $M S B$ and $M W T P$, we can next establish Proposition 2.

Proposition 2. The slope of the $M S B$ function is twice as steep as the slope of the MWTP.

Proof. See Appendix.

The equilibrium level of $N_{e}$ and its implication for the welfare of course depends on both the demand side for the technology, which we have just covered above, and on the supply side, which is the focus of the next section.

\section{Supply of teleworking technology}

\subsection{Perfect competition}

In this section we examine the pricing strategies of private (profit maximizing) and public (welfare maximizing) firms when supplying the teleworking technology. In particular, we are interested in the relative efficiency of private market outcomes, compared to the social optimum. The profit maximizing price is, for a given market structure, of course determined by the marginal willingness to pay through its impact on marginal revenue on the one hand, and marginal costs on the other. To reach the social optimum, a public provider should instead set a price that secures the equality of marginal social costs and marginal social benefits.

We assume that the marginal (social) cost of technology provision is zero. Besides simplifying the analysis, this assumption strengthens our finding that unrestricted supply of the teleworking technology might be marginally socially detrimental. The essential outcomes are not likely to change with the introduction of positive marginal costs. First, we will consider a market with perfect competition, where congestion is the single market friction. Then we introduce another market friction in combination to congestion: the existence of market power by a monopolist.

Under perfect competition the price is equal to zero marginal cost, so that in equilibrium all drivers are equipped with teleworking technology, i.e., $N_{e}^{P C}=\bar{N}$. The reason is that the $M W T P$, following the discussion in the previous section, is always positive (see Figure 4). The total social benefits under perfect competition $\left(T S B^{P C}\right)$ is then the integral of marginal social benefits in both low $\left(M S B_{L}\right)$ and high $\left(M S B_{H}\right)$ ranges of technology adoption: 


$$
T S B^{P C}=\int_{0}^{N_{e}^{\#}} M S B_{L} d N_{e}+\int_{N_{e}^{\#}}^{\bar{N}} M S B_{H} d N_{e}
$$

In contrast, a public firm sets the price and corresponding level of technology penetration, $N_{e}^{F B}$, such that the total social benefits are maximized, which yields:

$$
T S B^{F B}=\max _{N_{e}^{F B} \leq \bar{N}}\left(\int_{0}^{N_{e}^{\#}} M S B_{L} d N_{e}+\int_{N_{e}^{\#}}^{N_{e}^{F B}} M S B_{H} d N_{e}\right)
$$

Indeed, as used in (10), it is straightforward, albeit tedious and lengthy, to prove that, under zero marginal cost, the first-best level of technology penetration, $N_{e}^{F B}$, is always in the range $N_{e}^{\#}<N_{e}^{F B} \leq$ $\bar{N}$, implying that some equipped drivers arrive before $t^{*}{ }^{5}$ Thus, the socially optimal level of technology penetration $N_{e}^{F B}$ is then derived by equating $M S B_{H}$ to zero, where $M S B_{H}$ is defined by equation (A23) in Appendix:

$$
N_{e}^{F B}=\frac{\beta \Delta(2 \alpha-2 \beta+\Delta-\gamma) \bar{N}+\alpha(\beta+\gamma)\left(t^{*}-t_{S}\right)}{2(\alpha-\beta) \Delta(\beta+\gamma-\Delta)}
$$

If the corner solution $N_{e}^{F B}=\bar{N}$ holds, a competitive market provides the optimal outcome, as under perfect competition it is always the case that $N_{e}^{P C}=\bar{N}$. ${ }^{6}$ Otherwise, $T S B^{P C}<T S B^{F B}$, as the number of equipped drivers is too high. The tax would be required to bringing down the number of equipped drivers from $\bar{N}$ to $N_{e}^{F B}$, as the first-best price would be positive while under perfect competition it is zero (if marginal cost is zero). ${ }^{7}$

When comparing the outcomes of perfect competition and first-best, some results turn out to be cumbersome to present algebraically, so in this section we present results graphically, on the basis of numerical computations. In this model, numerical analysis can in fact be rather exhaustive, because all functions that are necessary for the analysis are dependent only on four parameters $\alpha, \beta, \gamma$ and $\Delta$.

\footnotetext{
${ }^{5} M S B$ is a discontinuous function which might cross marginal cost line of zero in two points: in the low and high ranges of penetration $N_{e}$. To see which gives the global maximum, one should compare the two integrals of $M S B$, one where the upper limit corresponds to the point of intersection in the low range, and one in the high range. The latter integral turns out to be always larger than the former. This also holds when MSB crosses the horizontal line only in the low range, while in the high range it ends up in the corner solution.

${ }^{6}$ Conditions when $N_{e}^{F B}=\bar{N}$ are defined below in equation (12).

${ }^{7}$ When marginal costs are positive and large enough, it might be possible that perfect competition will supply less than the optimal number of drivers, and then a subsidy is appropriate. After deriving equations of MWTP and $M S B$ it follows that if marginal costs are larger than $\Delta\left(t^{*}-t_{S}\right)-\frac{\beta \Delta(\gamma-\Delta) \bar{N}}{(\alpha+\Delta)(\beta+\gamma) s^{\prime}}$ then the competitive technology penetration level falls short of social optimal one, $N_{e}^{P C}<N_{e}^{F B}$.
} 
Without loss of generality, we may normalize the opportunity cost of being late at workplace as $\gamma=1$. The model restricts the effect from teleworking to $\Delta \in(0, \gamma)$. Most of the empirical literature suggests the relationship $\gamma>\alpha>\beta$ (e.g., Small, 1982). The relevant parameter space might then be shown as a cube with the edges $\alpha, \beta$ and $\Delta$, each of a length 1 (if desired, one could easily relax the constraint to allow both $\alpha$ and $\beta$ to be larger than $\gamma$ ). Without loss of generality, we normalize the overall number of drivers $\bar{N}$ to 100 ; and we set road capacity $s$ to 1 , so that the duration of the peak is 100 . This does not affect the results of interest. In subsequent computations we define the beginning of the day $t_{S}$ as the arrival time of the first driver when no teleworking is available (i.e., $t^{*}-t_{S}=\frac{\gamma}{\beta+\gamma} \frac{\bar{N}}{s}$ ). We can safely do this, because an introduction of teleworking always shifts the arrival window to later times. The reallocation of $t_{S}$ to an earlier stage increases the ideal utility $\Gamma_{e}$, as the time during which drivers are able to gain benefits from teleworking expands. But the increase takes place over a period where no one travels under any equilibrium, and we like to keep this "benefit" as small as possible. Changes in the end time $t_{F}$ do not affect the comparative performance of equilibria with and without teleworking, as this involves times of the day where only $W(t)$ matters for overall welfare, and this is not affected by the adaptation of the technology.

Figure 5 shows the domain of parameters values which make the perfect competition outcome of full penetration of the teleworking technology socially less desirable than the first-best outcome. The combinations of $\alpha, \beta$ and $\Delta$ within the meshed body are those for which $T S B^{P C}<T S B^{F B}$. The domain outside the meshed body in Figure 5 , in so far as it complies with the restriction $\alpha>\beta$, corresponds to the values where the competitive market generates the first-best outcome.

Figure 5. Parameter combinations that correspond to an above-optimal level of teleworking penetration under perfect competition $(\gamma=\mathbf{1})$

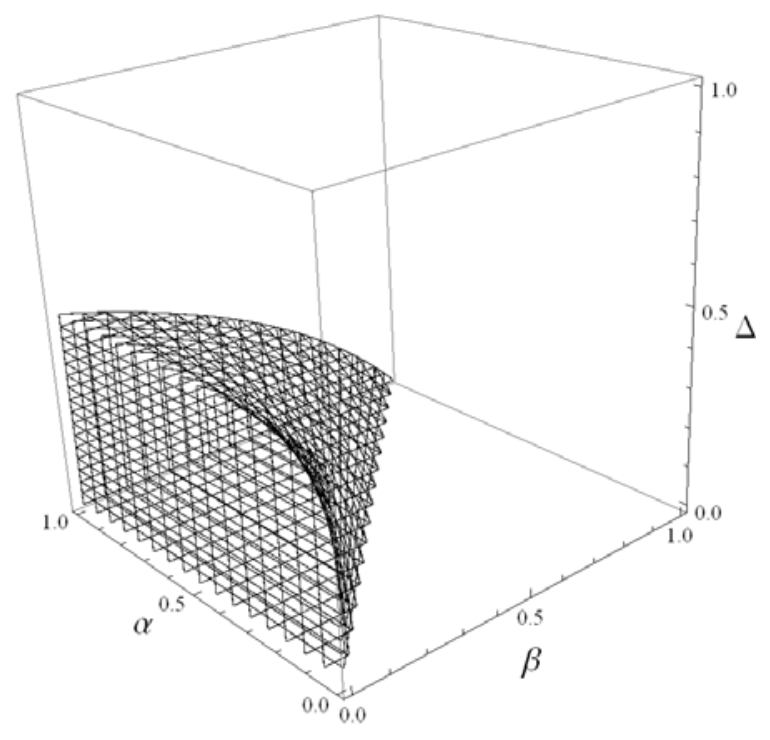


To better understand the conditions under which perfect competition could lead to an above-optimal penetration level, as in the meshed body in Figure 5, we compare the private benefits of the last unequipped driver when she gets equipped, with the negative externality on all other drivers she imposes. The condition for perfect competition to yield the first-best welfare gain, i.e., $\bar{N}=N_{e}^{P C}=N_{e}^{F B}$ when $N_{e}^{F B}=\frac{\beta(\Delta-\gamma-2 \beta)+\alpha(2 \beta+\gamma)}{2(\alpha-\beta)(\beta+\gamma-\Delta)} \bar{N}$, as follows from equation (11) when $t^{*}-t_{S}=\frac{\gamma}{\beta+\gamma} \frac{\bar{N}}{s}$, is:

$$
\Delta \geq \frac{\alpha-\beta}{2 \alpha-\beta}
$$

given that $\gamma=1$. When $\beta \rightarrow 0$, this condition becomes $\Delta>0.5$. If $\beta \rightarrow \alpha$, then it becomes $\Delta>0$. To see the intuition behind this pattern, note that the reason why perfect competition might not lead to the first-best outcome is that there is a difference between external costs that unequipped drivers impose on others (both equipped and unequipped), and the external costs from equipped drivers. That difference, conditional on $\Delta$, is small when $\beta \rightarrow \alpha$; and at the limit, when $\beta=\alpha$, it disappears completely. This happens because the slopes of isocost lines of early arrivals of both equipped and unequipped drivers become identical: $\frac{\beta}{\alpha}=\frac{\beta+\Delta}{\alpha+\Delta}=1$. This means that both groups trade-off travel delay and schedule delay costs identically, that the groups are not separated in time, and thus impose the same external costs on each other. An individual decision to become equipped then does not imply a change in the individual's external cost, so that as long as the individual herself benefits from doing so, also the net social welfare gain is positive. Even a small positive $\Delta$ is then enough to make full penetration of teleworking socially beneficial. However, when the difference in slopes of isocost lines between equipped and unequipped becomes larger ( $\alpha$ and $\beta$ diverge), implying larger differences in imposed negative externalities, a bigger gain $\Delta$ is required to "compensate" for larger net external costs imposed, and to make full penetration also socially optimal. At the limit, when $\beta \rightarrow 0$, the corresponding $\Delta$ is 0.5 . This explains the shape of the body in Figure 5. The value of $\gamma$ is irrelevant, as the difference between negative externalities of equipped and unequipped drivers under perfect competition is determined by the slopes of early arrivals.

\subsection{Private monopoly}

A private monopolistic provider is assumed to set the profit maximizing price. The profit of the monopolist $\left(\Pi^{M}\right)$, given zero marginal costs, and ignoring fixed costs, is the maximized integral of the marginal revenue $(M R)$ function, which itself directly follows from the MWTP function. For both ranges of levels of technology penetration, high and low, $M R$ is twice as steep as the MWTP. The monopolist's profit is: 


$$
\Pi^{M}= \begin{cases}\int_{0}^{N_{e}^{M}} M R_{L} d N_{e} & \mid 0 \leq N_{e}^{M} \leq N_{e}^{\#} \\ \int_{0}^{N_{e}^{\#}} M R_{L} d N_{e}+\int_{N_{e}^{\#}}^{N_{e}^{M}} M R_{H} d N_{e} & \mid N_{e}^{\#}<N_{e}^{M} \leq \bar{N}\end{cases}
$$

where the number of equipped drivers under monopoly is $N_{e}^{M}=\underset{N_{e}}{\operatorname{argmax}} \Pi^{M}$. In the numerical computations, we set the $M R$ function to zero, and check for the corresponding profit. Depending on the parameter values, $M R$ might cross in both ranges of technology penetration (high and low), and then we numerically check for the largest resulting profit. The corresponding total social benefits $\left(T S B^{M}\right)$ is then the integral of the marginal social benefits, with $N_{e}^{M}$ as the upper limit:

$$
T S B^{M}= \begin{cases}\int_{0}^{N_{e}^{M}} M S B_{L} d N_{e} & 0 \leq N_{e}^{M} \leq N_{e}^{\#} \\ \int_{0}^{N_{e}^{\#}} M S B_{L} d N_{e}+\int_{N_{e}^{\#}}^{N_{e}^{M}} M S B_{H} d N_{e} & N_{e}^{\#}<N_{e}^{M} \leq \bar{N}\end{cases}
$$

Because $M R$ and $M S B$ are not equal, the monopolist matches the first-best outcome only when achieving full penetration; i.e., when monopolist ends up in the corner solution of $N_{e}^{M}=\bar{N}$. This requires $M R$ to be high enough; for instance, when $\Delta$ is large.

As observed in Section 3, MSB always exceeds $M R$; therefore, the private monopolist will never supply more than the optimal number of drivers with the teleworking technology. One underlying reason is that the private provider internalizes the negative external effects that its customers impose upon one another. The positive externalities of teleworking to unequipped drivers are, however, left outside the monopolistic pricing rule while it would reduce the socially optimal price, implying that the profitmaximizing price exceeds the welfare-maximizing price also for a reason different from the classic demand-related mark-up. We can summarize our findings on the level of penetration under different market forms in Proposition 3.

Proposition 3. Given zero marginal costs, equilibrium levels of technology penetration under different market forms relate to each other in the following manner: $N_{e}^{M} \leq N_{e}^{F B} \leq N_{e}^{P C}$.

Proof. See Appendix.

Next, in Figure 6 we compare the welfare outcomes of perfect competition versus the private monopoly. The combination of $\alpha, \beta$ and $\Delta$ within the meshed body are those for which $T S B^{P C}<T S B^{M}$, so monopoly produces a higher social welfare than perfect competition does. The parameter space outside 
the meshed body, insofar as $\alpha>\beta$, corresponds to the values where either perfect competition outperforms monopoly, or where both yield the same outcome in terms of social welfare.

For a sizable parameter space, a monopoly market leads to a higher social welfare than perfect competition. The body of Figure 6 lies entirely within that of Figure 5. That is: total social benefits under monopoly can be larger than under perfect competition only if perfect competition itself is not the firstbest outcome. If the strict inequality $N_{e}^{M}<N_{e}^{F B}<N_{e}^{P C}$ holds, the monopoly level of penetration might be "closer" (in terms of welfare) to the first-best level than perfect competition, as the latter always produces $\bar{N}$. Figure 6 shows that perfect competition is particularly "harmful" in terms of oversupply when the differences between external effects of unequipped on equipped vs. equipped on themselves is large; i.e., when $\beta$ diverges from $\alpha$. Not shown explicitly in Figure 6 is the subset of parameter values where the monopolist prefers to be in the low range of penetration; i.e. $0 \leq N_{e}^{M} \leq N_{e}^{\#}$. That area touches the one shown in Figure 6, and is located in the bottom part (low $\Delta$ ), in the corner with high $\alpha$ and $\beta$ (but $\alpha>\beta$ ). There, the resulting $T S B^{M}$ is so low, that oversupply of perfect competition is socially preferable.

Figure 6. Parameter combinations that have a larger total social welfare under monopoly than under perfect competition $(\gamma=1)$

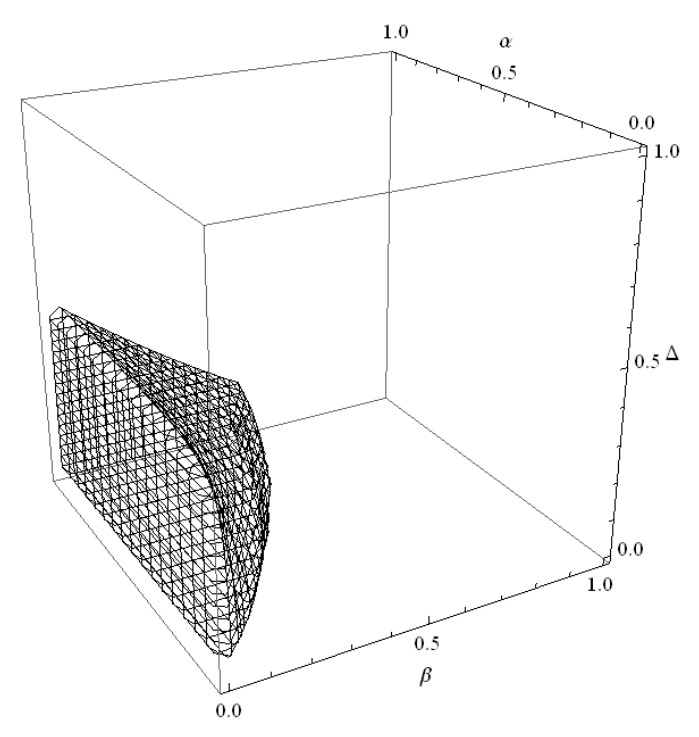

Small (1982) was the first to provide empirical values for the parameters $\alpha, \beta$ and $\gamma$, which suggest that their relative values approximately satisfy: $\gamma=2 \alpha=4 \beta$. This combination of parameter values turns out to be within the body displayed in the Figure 6 for relatively low values of $\Delta$. This suggests that monopolistic supply of the teleworking technology might be more attractive than competitive supply for moderate values of $\Delta$, which in turn would reflect limited attractiveness of working at home with the technology, compared to being at work. For example, for the parameter values $\gamma=1, \alpha=0.5$, 
$\beta=0.25$ and $\Delta=0.125$, the equilibrium levels of technology penetration and corresponding total welfare levels under, respectively, perfect competition, welfare maximization and monopoly are $N_{e}^{P C}=100, N_{e}^{F B}=72.2, N_{e}^{M}=61.1$ and $T S B^{P C}=375, T S B^{F B}=418.4$ and $T S B^{M}=411.5$.

\section{Teleworking with the first-best road toll}

We have now established how, in the presence of congestion, the use of the teleworking technology by equipped drivers causes externalities for others. A consequence is that it may not be optimal to supply the technology at marginal production cost; zero, in our case. The second-best distortion that is responsible for this, is the unpriced congestion at the bottleneck. A relevant question is whether the externality in the consumption of the technology, and hence the optimal deviation from marginal cost pricing for the purchase, vanishes when congestion at the bottleneck is optimally priced.

A central result in the literature on Vickrey's dynamic bottleneck model is that waiting time is a pure social loss, which can be fully eliminated through optimal time-varying pricing (Vickrey, 1969; Arnott, de Palma and Lindsey, 1993). The social optimum is achieved by levying a first-best time-dependent road toll that exactly equals the travel delay costs in the no-toll equilibrium, at each moment of arrival. Thus, instead of waiting in the queue, drivers pay a toll and incur no waiting time. With homogeneous drivers, the generalized travel price thus remains unchanged, compared to the no-toll case considered earlier. But from the social viewpoint, a toll is not a cost component, but a welfare neutral monetary transfer from road users to government. The welfare gain from first-best pricing is therefore equal to the total toll revenues, and therewith to the total savings in travel delay cost. For more in-depth discussion of the model with pricing we refer to, among others, Arnott, de Palma and Lindsey (1993).

Figure 7 shows the optimal toll schedule for homogeneous unequipped drivers. The schedule depends entirely on the parameters $\beta, \gamma, s$ and $\bar{N}$. Note that the peak starts at the same time $t_{q}$ as in the no-toll case, because the very first and the very last driver in both toll and no-toll regimes incur schedule delay costs only, which should be equalized also in the optimum. The generalized price of travel is therefore also equal to that in the no-toll equilibrium. The toll schedule has slopes $\beta$ and $-\gamma$ to keep the price constant over time without a queue, and reaches its maximum at the preferred arrival time $t^{*}$.

Figure 7 represents the isoprice function, and thus resembles Figure 2 for the no-toll case, the only difference being the slopes. These are $\beta$ and $-\gamma$ with first-best pricing, and $\frac{\beta}{\alpha}$ and $-\frac{\gamma}{\alpha}$ in the no-toll equilibrium. This difference only reflects the different units used in the vertical dimension (money in Figure 7 versus time in Figure 2).

It is now straightforward to repeat the entire analysis from the section 3, under the new conditions of optimal road pricing. The slopes of the isoprice curves for early arrivals and for the late ones are, respectively, $\beta$ and $-\gamma$ for unequipped drivers, and $\beta+\Delta$ and $-(\gamma-\Delta)$ for equipped ones. The isoprice slope is flatter for equipped drivers for late arrivals and steeper for early ones. Thus, a temporal 
separation in arrival times of equipped and unequipped drivers occurs in qualitatively the same manner as in the no-toll case: unequipped travelers go first, equipped ones go last.

Figure 7. Isoprice function of the homogeneous drivers

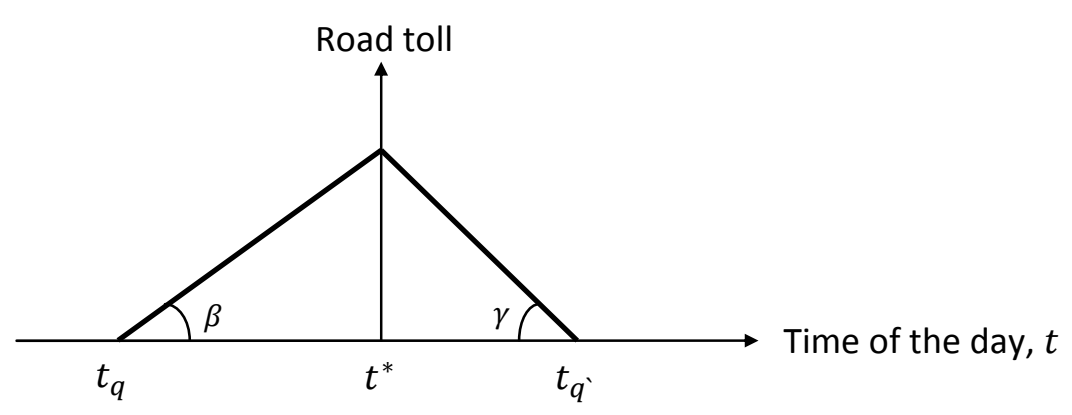

Furthermore, we can calculate $M W T P$ and $M S B$ in the same way as described in, respectively, subsections 3.1 and 3.2. Because the order of arrival at work is the same as in no-toll case, the revenues from the toll collection are exactly 50 percent of the combined generalized travel costs that all drivers incur (as is true in the conventional bottleneck model with homogeneous users). Thus, the total social costs are cut by half. The MWTP and MSB functions are now identical:

$$
M W T P=M S B=\Delta\left(t^{*}-t_{S}\right)+\frac{\beta \Delta}{\beta+\gamma} \frac{\bar{N}}{S}-\frac{\Delta(\beta+\gamma-\Delta)}{\beta+\gamma} \frac{N_{e}}{s}
$$

The equality of $M W T P$ and $M S B$ implies that the teleworking technology becomes a conventional good when the congestion externality is perfectly internalized by the first-best road toll. That is, no policy interventions are required to bring $M S B$ equal to the marginal costs under perfect competition. Equilibrium technology penetration under perfect competition corresponds to socially optimal one, and is derived by setting equation (15) to zero, which results in $N_{e}^{P C}=N_{e}^{F B}=\bar{N}$. And under monopoly, the regular overpricing due to market power occurs. As usual for a monopolist with linear demand and constant marginal cost, the equilibrium share of equipped drivers is exactly half of that under the firstbest, i.e., $N_{e}^{M}=0.5 \bar{N}$, as the slope of MR function is twice as steep as that of MWTP.

Finally, note that for both low $\left(0 \leq N_{e} \leq N_{e}^{\#}\right)$, and high numbers $\left(N_{e}^{\#}<N_{e} \leq \bar{N}\right)$ of equipped drivers, the functions are the same, i.e., there is now no kink in $M W T P$, and no discontinuity in the MSB. The reason why the kink in MWTP disappears is that equipped drivers impose an identical unpriced "net" externality, i.e., in excess of the toll level, namely zero under first-best pricing, whether they arrive after or before $t^{*}$. It was the difference in unpriced externalities imposed by early versus late equipped 
drivers that caused the kink for the no-toll case, and this difference now no longer exists with optimal pricing.

\section{Summary and conclusions}

We investigated the welfare effects from teleworking becoming available for a congested bottleneck, using Vickrey's (1969) dynamic bottleneck model. Teleworking was modeled as an increase in the utility that a person derives from being at home, sufficiently small to keep commuting worthwhile. We derived the marginal willingness to pay for teleworking as the difference in benefit that a driver attains when being equipped with teleworking-enabling technology, compared to being unequipped. Getting the possibility of teleworking creates differences in the utility parameters of otherwise homogeneous drivers, and consequently affects their dynamic travel behavior.

As compared to unequipped drivers, an equipped driver values being at home relatively high, while late arrival becomes relatively less problematic, as she can "compensate" being late by working from home. Thus, we show that equipped drivers depart from home to be "at the end of the peak" to gain the most out of teleworking. But the rising number of the equipped drivers makes the end of traffic jam increasingly late, and thus diminishes teleworking benefits and therewith the marginal willingness to pay for it. As the marginal external costs differ between equipped and unequipped drivers, the decision to become equipped influences travel costs of all other drivers.

We derive generalized travel costs for both equipped and unequipped drivers, and the total social benefits of teleworking as a function of the number of equipped drivers. The optimal level of technology penetration is then such that the marginal social benefit is equal to the marginal social cost. We compared the relative efficiency of private market outcomes, under monopoly and perfect competition, to the social optimum. Finally, we examined the effect of teleworking on travel costs when the congestion externality is internalized using the time-dependent first-best road toll.

We find that even costless teleworking might have an adverse marginal effect on social welfare, when a certain level of technology penetration is reached, due to the negative externality it creates. The very first unequipped driver who becomes equipped prefers to be the only one teleworking, as equipped drivers impose higher external travel cost on one another than unequipped impose on them. The more people are teleworking, therefore, the lower the benefits of teleworking for each individual equipped driver. The remaining unequipped drivers enjoy positive effects of teleworking: the negative externality of equipped drivers on unequipped ones is lower than what unequipped drivers impose upon one another. Although full penetration of costless teleworking is always socially more beneficial than no teleworking at all, there exists an optimal degree of driver heterogeneity. An increase in the number of equipped drivers above that level lowers social welfare.

Our results show that private monopolistic supply of a teleworking technology might yield higher social welfare than perfect competition does. Under perfect competition, with zero marginal cost, all drivers 
are teleworking, as the endogenous marginal willingness to pay for teleworking is always positive. A full penetration might, however, be socially excessive. At the same time, a monopolist charges a mark-up, while taking into account the negative effects its customers impose one each other, but ignoring the positive effects on unequipped drivers. The level of penetration under monopoly could consequently be below the optimal level. We identified the conditions under which the monopoly outcome is "closer" to optimal, from a social welfare viewpoint, than that of perfect competition.

A policy that internalizes the congestion externality would also avoid distortions from the changes in externalities resulting from the purchase of the technology. Time-dependent first-best road toll achieves this, and makes the teleworking technology a "conventional good", which does not require any policy intervention when supplied under perfectly competitive conditions.

To the best of our knowledge, this paper is the first to model the effect of (part-day) teleworking on generalized travel costs. We have used a conventional linearized scheduling model, as considered by Vickrey (1969), Small (1982), Arnott, de Palma and Lindsey (1993), but we assumed it stems from preferences of being at home and being at work in a way as described by Vickrey (1973) and, later, by Tseng and Verhoef (2008). We found this framework suits well for the analysis and yields interesting insights.

There is ample scope for further research on the effects of teleworking on travel within the considered framework. Among the possible extensions are a consideration of initial driver heterogeneity (a tentative discussion see in Appendix); variation in teleworking technology; and more complex road networks allowing for an explicit consideration of spatial and network effects, in addition to the temporal dimension considered here. It might also be interesting to incorporate other effects of teleworking besides those on travel costs, such as effects on productivity, work-life balance, etc., to get the full picture of the overall effect of teleworking on welfare.

\section{References}

Arnott, R., A. de Palma, and R. Lindsey, "A Structural Model of Peak-Period Congestion: A Traffic Bottleneck with Elastic Demand," American Economic Review 83 (1993), 161-179.

— - _ - and — - "The Welfare Effects of Congestion Tolls with Heterogeneous Commuters," Journal of Transport Economics and Policy 28 (1994), 139-161.

—, - and — - "Information and Usage of Free-Access Congestible Facilities with Stochastic Capacity and Demand," International Economic Review 37 (1996), 181-203.

De Palma, A., and R. Lindsey, "Information and Usage of Congestible Facilities under Different Pricing Regimes," Canadian Journal of Economics 31 (1998), 666-692. 
Emmerink, R.H.M., E.T. Verhoef, P. Nijkamp, and P. Rietveld, "Information Policy in Road Transport with Elastic Demand: Some Welfare Economic Considerations," European Economic Review 42 (1998a), 71-95.

$\longrightarrow,-, \ldots$, and _ _ - "Information Effects in Transport with Stochastic Capacity and Uncertainty Costs," International Economic Review 39 (1998b), 89-110.

Eurofound, Third European Survey on Working Conditions 2000 (Dublin, 2005).

_ Teleworking in the European Union (Dublin, 2010).

Glaeser, E.L., Cities, Agglomeration and Spatial Equilibrium (New York: Oxford University Press, 2008)

Haddad, H., G. Lyons, and K. Chatterjee, "An Examination of Determinants Influencing the Desire for and Frequency of Part-Day and Whole-Day Homeworking," Journal of Transport Geography 17 (2009), 124-133.

Pigou, A.C., Wealth and Welfare (London: Macmillan, 1920).

Rhee, H.-J., "Home-Based Telecommuting and Commuting Behavior," Journal of Urban Economics 63 (2008), 198-216.

Safirova, E., "Telecommuting, Traffic Congestion, and Agglomeration: A General Equilibrium Model," Journal of Urban Economics 52 (2002), 26-52.

Small, K.A., "The Scheduling of Consumer Activities: Work Trips," American Economic Review 72 (1982), 467-479.

—

Tseng, Y.-Y., and E.T. Verhoef, "Value of Time by Time of Day: A Stated-Preference Study," Transportation Research Part B: Methodology 42 (2008), 607-618.

Vickrey, W.S., "Congestion Theory and Transport Investment," American Economic Review (Papers and Proceedings) 59 (1969), 251-260.

_- "Pricing, Metering, and Efficiently Using Urban Transportation Facilities," Highway Research Record 476 (1973), 36-48.

\section{Appendix}

Lemma 1. The generalized travel costs for unequipped drivers $P_{u}$ is decreasing when the share of equipped drivers $N_{e}$ rises, i.e., $\frac{\partial P_{u}}{\partial N_{e}}<0$. 
Proof. The proof consists of two parts - the first part proves Lemma 1 for the "low" range of $N_{e}$, i.e., $0 \leq N_{e} \leq N_{e}^{\#}$, the second part proves Lemma 1 for the "high" range, i.e., $N_{e}^{\#}<N_{e} \leq \bar{N}$.

For the first part, to calculate equilibrium cost levels as a function of $N_{e}$, we indicate the travel delay that the first arrived equipped driver incurs as $X$, while $Y$ gives the absolute difference between $X$ and the travel delay of a driver who arrives at work at time $t^{*}$. We can write $X$ and $Y$ as follows:

(A1) $\quad X+Y=\frac{\beta}{\alpha} \frac{N_{\mathcal{u}}^{E}}{s}=\frac{\beta}{\alpha} \frac{\bar{N}-N_{e}-N_{\mathcal{u}}^{L}}{s}$

(A2) $\quad X=\frac{\gamma-\Delta}{\alpha+\Delta} \frac{N_{e}}{s}$

(A3) $\quad Y=\frac{\gamma}{\alpha} \frac{N_{u}^{L}}{s}$

These equalities can easily be verified in Figure 3. After substituting (A2) and (A3) into (A1), we can express $\frac{N_{u}^{L}}{s}$ (i.e., the duration of the interval where unequipped drivers arrive after $t^{*}$ ) and $\frac{N_{u}^{E}}{s}$ (i.e., the duration of the peak before $t^{*}$ ) as a function of $N_{e}$ :

$$
\frac{N_{u}^{L}}{s}=\frac{\beta}{\beta+\gamma} \frac{\bar{N}}{s}-\frac{(\alpha+\Delta) \beta+\alpha(\gamma-\Delta)}{(\alpha+\Delta)(\beta+\gamma)} \frac{N_{e}}{s}
$$

(A5) $\quad \frac{N_{u}^{E}}{s}=\frac{\gamma}{\beta+\gamma} \frac{\bar{N}}{s}-\frac{\Delta(\alpha+\gamma)}{(\alpha+\Delta)(\beta+\gamma)} \frac{N_{e}}{s}$

Multiplying (A5) by $\beta$, we get the generalized travel costs of unequipped drivers as a function of $N_{e}$ (for $\left.0 \leq N_{e} \leq N_{e}^{\#}\right)$ :

(A6) $\quad P_{u}=\frac{N_{u}^{E}}{s} \beta=\frac{\gamma \beta}{\beta+\gamma} \frac{\bar{N}}{s}-\frac{\Delta \beta(\alpha+\gamma)}{(\alpha+\Delta)(\beta+\gamma)} \frac{N_{e}}{s}$

Since all Greek characters symbolize positive parameters, and $s$ is positive too, equation (A6) shows that $\frac{\partial P_{u}}{\partial N_{e}}<0$, for the "low" range of $N_{e}$.

The logic of the derivation of $P_{u}$ stays the same for the "high" range, i.e., $N_{e}^{\#}<N_{e} \leq \bar{N}$.

Let $N_{e}^{E}$ be the number of equipped drivers arriving early, and $N_{e}^{L}$ the number arriving late, so that $N_{e}=N_{e}^{E}+N_{e}^{L}$. For the present case $N_{e}^{\#}<N_{e} \leq \bar{N}$, we can express $X$ and $Y$, as shown in Figure A1, as: 
(A7) $\quad X+Y=\frac{\gamma-\Delta}{\alpha+\Delta} \frac{N_{e}^{L}}{s}=\frac{\gamma-\Delta}{\alpha+\Delta} \frac{\bar{N}-N_{u}^{E}-N_{e}^{E}}{s}$

(A8) $\quad X=\frac{\beta}{\alpha} \frac{N_{u}^{E}}{s}=\frac{\beta}{\alpha} \frac{\bar{N}-N_{e}^{E}-N_{e}^{L}}{s}$

(A9) $\quad Y=\frac{\beta+\Delta}{\alpha+\Delta} \frac{N_{e}^{E}}{s}$

Figure A1. Isocost functions of the heterogeneous drivers, if equipped drivers arrive at work late and early

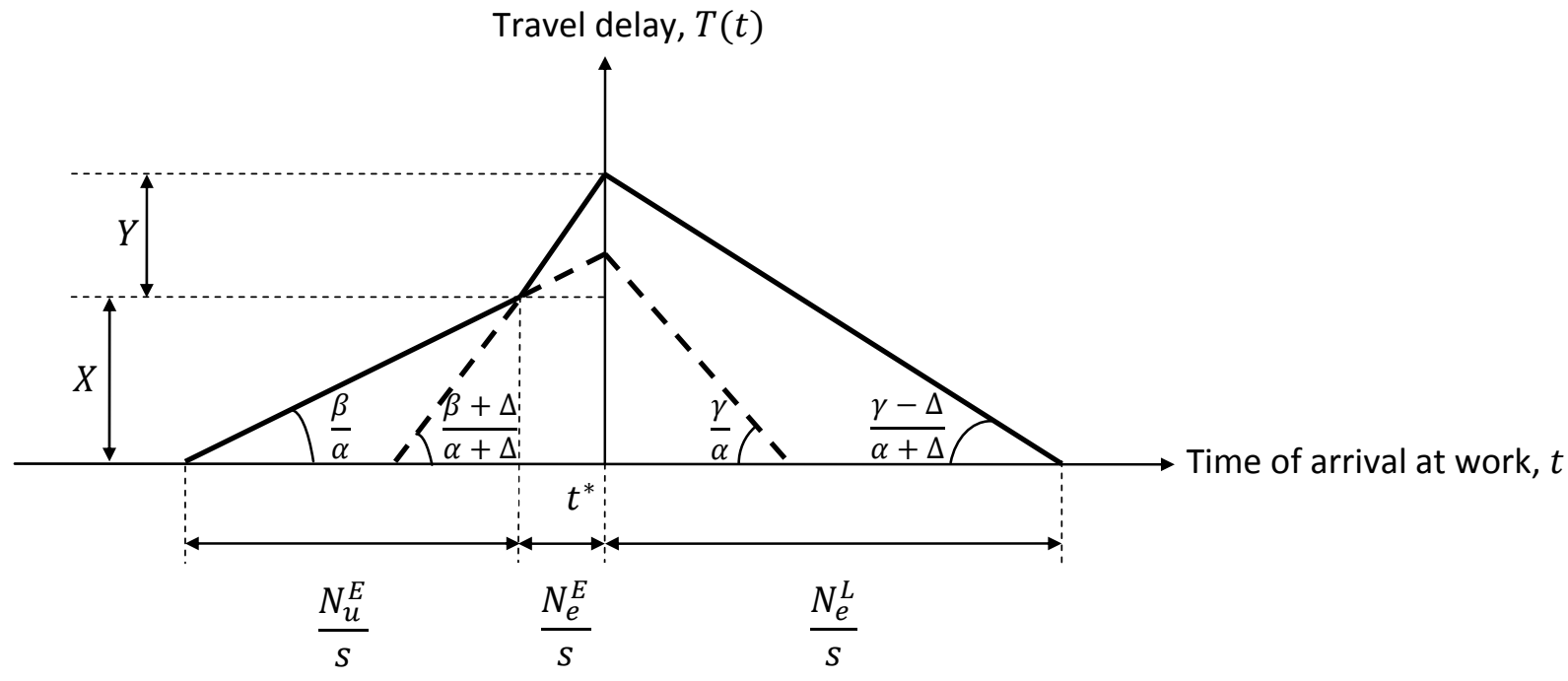

After substituting (A8) and (A9) into (A7), we derive $\frac{N_{e}^{E}}{s}$ (i.e., the duration of the interval when equipped drivers arrive early) and $\frac{N_{e}^{L}}{s}$ (i.e., the duration of the peak after $\left.t^{*}\right)$ :

(A10) $\frac{N_{e}^{E}}{s}=-\frac{(\alpha+\Delta) \beta}{\alpha(\beta+\gamma)} \frac{\bar{N}}{s}+\frac{(\alpha+\Delta) \beta+\alpha(\gamma-\Delta)}{\alpha(\beta+\gamma)} \frac{N_{e}}{s}$

(A11) $\frac{N_{e}^{L}}{s}=\frac{(\alpha+\Delta) \beta}{\alpha(\beta+\gamma)} \frac{\bar{N}}{s}+\frac{(\alpha-\beta) \Delta}{\alpha(\beta+\gamma)} \frac{N_{e}}{s}$

The generalized cost for unequipped drivers, become, respectively: 
(A12) $\quad P_{u}=\left(\frac{\bar{N}}{s}-\frac{N_{e}^{L}}{s}\right) \beta=\left(\frac{\bar{N}}{s}-\frac{(\alpha+\Delta) \beta}{\alpha(\beta+\gamma)} \frac{\bar{N}}{s}-\frac{(\alpha-\beta) \Delta}{\alpha(\beta+\gamma)} \frac{N_{e}}{s}\right) \beta$

Equation (A12) shows that $\frac{\partial P_{u}}{\partial N_{e}}<0$, for the "high" range of $N_{e}$ (recall that $\alpha>\beta$ ).

Lemma 2. The generalized travel costs of equipped drivers $P_{e}$ is increasing when the share of equipped drivers $N_{e}$ rises, i.e., $\frac{\partial P_{e}}{\partial N_{e}}>0$.

Proof. Using equations (A4) and (A11) we define the generalized travel costs of equipped drivers $P_{e}$, for, respectively, the "low" and "high" ranges of $N_{e}$ as follows:

$$
P_{e}=\left(\frac{N_{u}^{L}}{s}+\frac{N_{e}}{s}\right)(\gamma-\Delta)=\frac{\beta(\gamma-\Delta)}{\beta+\gamma} \frac{\bar{N}}{s}+\frac{\Delta(\gamma-\Delta)(\alpha+\gamma)}{(\alpha+\Delta)(\beta+\gamma)} \frac{N_{e}}{s} \quad \mid 0 \leq N_{e} \leq N_{e}^{\#}
$$

$$
P_{e}=\frac{N_{e}^{L}}{s}(\gamma-\Delta)=\frac{(\gamma-\Delta)(\alpha+\Delta) \beta}{\alpha(\beta+\gamma)} \frac{\bar{N}}{s}+\frac{(\alpha-\beta) \Delta(\gamma-\Delta)}{\alpha(\beta+\gamma)} \frac{N_{e}}{s} \quad \mid N_{e}^{\#}<N_{e} \leq \bar{N}
$$

The lemma follows immediately from (A13) and (A14), given that $\alpha>\beta$ and $\gamma>\Delta$.

Proposition 1. MWTP is negatively related to number of equipped drivers $N_{e}$, so if more drivers are equipped, an additional individual driver is willing to pay less for teleworking technology, i.e., $\frac{\partial M W T P}{\partial N_{e}}<$ 0

Proof. For the "low" range of equipped drivers, we use equations (A6) and (A13) to derive utility of, respectively, unequipped and equipped drivers:

$$
\begin{aligned}
& U_{u}=\Gamma_{u}-P_{u}=\alpha\left(t^{*}-t_{S}\right)+(\alpha+\gamma)\left(t_{F}-t^{*}\right)-\frac{\gamma \beta}{\beta+\gamma} \frac{\bar{N}}{s}+\frac{\Delta \beta(\alpha+\gamma)}{(\alpha+\Delta)(\beta+\gamma)} \frac{N_{e}}{s} \\
& U_{e}=\Gamma_{e}-P_{e}=(\alpha+\Delta)\left(t^{*}-t_{S}\right)+(\alpha+\gamma)\left(t_{F}-t^{*}\right)-\frac{\beta(\gamma-\Delta)}{\beta+\gamma} \frac{\bar{N}}{s}-\frac{\Delta(\gamma-\Delta)(\alpha+\gamma)}{(\alpha+\Delta)(\beta+\gamma)} \frac{N_{e}}{s}
\end{aligned}
$$

We plug in equations (A15) and (A16) into equation (5) to derive $M W T P$ when $0 \leq N_{e} \leq N_{e}^{\#}$ : 
(A17) $\quad M W T P=U_{e}-U_{u}=\Gamma_{e}-P_{e}-\Gamma_{u}+P_{u}=\Delta\left(t^{*}-t_{s}\right)+\frac{\beta \Delta}{\beta+\gamma} \frac{\bar{N}}{s}-\frac{\Delta(\beta+\gamma-\Delta)(\alpha+\gamma)}{(\alpha+\Delta)(\beta+\gamma)} \frac{N_{e}}{s}$

Equation (A17) shows that $\frac{\partial M W T P}{\partial N_{e}}<0$ (recall that $\left.\gamma>\Delta\right)$.

For the "high" range of equipped drivers, we use equations (A12) and (A14) to derive utility of, respectively, unequipped and equipped drivers:

(A18)

$$
\begin{aligned}
& U_{u}=\Gamma_{u}-P_{u}=\alpha\left(t^{*}-t_{S}\right)+(\alpha+\gamma)\left(t_{F}-t^{*}\right)-\left(\frac{\bar{N}}{s}-\frac{(\alpha+\Delta) \beta}{\alpha(\beta+\gamma)} \frac{\bar{N}}{s}-\frac{(\alpha-\beta) \Delta}{\alpha(\beta+\gamma)} \frac{N_{e}}{s}\right) \beta \\
& U_{e}=\Gamma_{e}-P_{e}=(\alpha+\Delta)\left(t^{*}-t_{S}\right)+(\alpha+\gamma)\left(t_{F}-t^{*}\right)-\frac{(\gamma-\Delta)(\alpha+\Delta) \beta}{\alpha(\beta+\gamma)} \frac{\bar{N}}{s}-\frac{(\alpha-\beta) \Delta(\gamma-\Delta)}{\alpha(\beta+\gamma)} \frac{N_{e}}{s}
\end{aligned}
$$

We plug in equations (A18) and (A19) into equation (5) to derive $M W T P$ when $N_{e}^{\#}<N_{e} \leq \bar{N}$ :

$$
M W T P=U_{e}-U_{u}=\Gamma_{e}-P_{e}-\Gamma_{u}+P_{u}=\Delta\left(t^{*}-t_{S}\right)-\frac{\beta \Delta(\beta-\alpha+\gamma-\Delta))}{\alpha(\beta+\gamma)} \frac{\bar{N}}{s}-\frac{\Delta(\beta+\gamma-\Delta)(\alpha-\beta)}{\alpha(\beta+\gamma)} \frac{N_{e}}{s}
$$

Equation (A20) shows that $\frac{\partial M W T P}{\partial N_{e}}<0$ (recall that $\alpha>\beta$ and $\gamma>\Delta$ ).

Proposition 2. The slope of the $M S B$ function is twice as steep as the slope of the MWTP.

Proof. Let TSC be the total social costs, $\varphi=\frac{\partial P_{u}}{\partial N_{e}}$, and $\mu$ and $\theta$ are constants to be defined below. Following equations (A6), (A12), (A13), (A14) we can write the difference in generalized travel costs as a function of the number of equipped drivers $N_{e}$ :

$$
P_{u}-P_{e}=\mu \bar{N}-\theta N_{e}, \text { with }
$$

$\mu= \begin{cases}\frac{\beta \Delta}{(\beta+\gamma) s}, & \mid 0 \leq N_{e} \leq N_{e}^{\#} \\ \frac{\beta \Delta(\alpha-\beta-\gamma+\Delta)}{\alpha(\beta+\gamma) s}, & \mid N_{e}^{\#}<N_{e} \leq \bar{N}\end{cases}$ 
$\theta= \begin{cases}\frac{\Delta(\alpha+\gamma)(\beta-\Delta+\gamma)}{(\alpha+\Delta)(\beta+\gamma) s}, & \mid 0 \leq N_{e} \leq N_{e}^{\#} \\ \frac{\Delta(\alpha-\beta)(\beta-\Delta+\gamma)}{\alpha(\beta+\gamma) s}, & \mid N_{e}^{\#}<N_{e} \leq \bar{N}\end{cases}$

For both "low" and "high" ranges of the technology penetration we can write MWTP as:

(A22) $M W T P=U_{e}-U_{u}=\left(\Gamma_{e}-P_{e}\right)-\left(\Gamma_{u}-P_{u}\right)=\Gamma_{e}-\Gamma_{u}+P_{u}-P_{e}=\Delta\left(t^{*}-t_{S}\right)+\mu \bar{N}-\theta N_{e}$.

$T S C$ is the sum of the costs of equipped and unequipped drivers:

$T S C=N_{u} P_{u}+N_{e} P_{e}=\left(\bar{N}-N_{e}\right) P_{u}+N_{e} P_{e}=\bar{N} P_{u}+N_{e}\left(P_{e}-P_{u}\right)=\bar{N} P_{u}+N_{e}\left(\theta N_{e}-\mu \bar{N}\right)$

Note that by definition $M S B=-\frac{\partial T S C}{\partial N_{e}}+\left(\Gamma_{e}-\Gamma_{u}\right)$; that leads to:

$-M S B=\frac{\partial T S C}{\partial N_{e}}-\left(\Gamma_{e}-\Gamma_{u}\right)=\bar{N} \frac{\partial P_{u}}{\partial N_{e}}+2 \theta N_{e}-\mu \bar{N}-\Delta\left(t^{*}-t_{S}\right)=\varphi \bar{N}+2 \theta N_{e}-\mu \bar{N}-\Delta\left(t^{*}-t_{S}\right)$

From equations (A6) and (A12) we see that $\varphi$ is a (negative) constant which does not depend on $N_{e}$, hence the slope of the $M S B$ function is twice as steep as the slope of the MWTP:

$$
M S B=\Delta\left(t^{*}-t_{S}\right)+(\mu-\varphi) \bar{N}-2 \theta N_{e}
$$

Proposition 3. Given zero marginal costs, equilibrium levels of technology penetration under different market forms relate to each other in the following manner: $N_{e}^{M} \leq N_{e}^{F B} \leq N_{e}^{P C}$.

Proof. The MWTP of the last unequipped driver who becomes equipped is positive, as follows from equation (A20), i.e., $\left(M W T P \mid N_{e}=\bar{N}\right)>0$. Thus, under zero marginal costs, an equilibrium level of technology penetration under the perfect competition is $\bar{N}$, as MWTP function is monotonously decreasing with respect to $N_{e}$.

$N_{e}^{F B}$ can be equal to $\bar{N}$, as we showed in Figure 5, when $\Delta \geq \frac{\alpha-\beta}{2 \alpha-\beta}$ (this holds if the start of the morning period is $\left.t_{S}=t^{*}-\frac{\gamma}{\beta+\gamma} \frac{\bar{N}}{S}\right)$, otherwise $N_{e}^{F B}<\bar{N}$. The $M R$ function as well as the MSB function, is twice as steep as the slope of the MWTP (as follows from the Proposition 2). However, the intercept of MSB function is larger than that of $M R$ function (compare equations (A22) and (A23)). That makes $M R$ function to cross the horizontal line to the left of $M S B$ function, implying $N_{e}^{M}<N_{e}^{F B}$ (unless it is a corner solution when $N_{e}^{M}=N_{e}^{F B}=\bar{N}$ ).

\section{Discussion of initial driver heterogeneity}

Modeling driver heterogeneity in the bottleneck model is not an easy task, and it is not straightforward to predict how the existence of initial heterogeneity would affect the impacts of endogenous 
heterogeneity through the purchase of the teleworking technology considered in this paper. We can make some speculative observations though, considering different types of heterogeneity that could exist. But we hasten to say that a formal analysis, which is outside the scope of this paper for reasons of space, would be required to make more definite statements.

If there would be heterogeneity with respect to the desired time of arrival, $t^{*}$, we would expect that as long as the distribution is sufficiently dense so that a single peak period with congestion always arises a sufficient condition for which is that the cumulative desired arrivals exceeds the cumulative actual arrivals between two moments in time - not much will change. For the basic bottleneck model, such heterogeneity would leave the queuing pattern unchanged, while reducing the schedule delay cost (e.g., Small and Verhoef, 2007). We would expect a similar effect in the current model, especially if the time window of desired arrival times is between the first and the last arrival both before and after the technology has become available.

A second type of heterogeneity would involve initial differences in the time-related shadow prices, a problem considered by, for example, Arnott, de Palma and Lindsey (1994) for discrete distributions, and van den Berg and Verhoef (2011) for continuous ones. To put some structure on the problem, it is helpful here to distinguish between "proportional heterogeneity", where all shadow prices vary across individuals but their ratios are equal for all (e.g., an $\alpha$ twice as high also means a $\beta$ twice as high, and so on), and "ratio heterogeneity", where ratios of shadow prices do vary across travelers, usually achieved by letting only $\alpha$ to vary.

For proportional heterogeneity, it seems reasonable to assume that also $\Delta$ would vary in the same way over individuals as $\alpha, \beta$ and $\gamma$. Call the factor of proportionality $\mu$. Under this type of heterogeneity, without tolling, travelers would pool, and not drive separated in time, other than as caused by being equipped with the teleworking technology or not, since the equilibrium slopes of the indifference functions as shown in Figures 2 and 3 would be the same for all. In that case, it seems that the willingness to pay for the first driver to become equipped (which is equal to $\frac{\Delta \bar{N}}{s}$, if $t^{*}-t_{S}=\frac{\gamma}{\beta+\gamma} \frac{\bar{N}}{s}$ ), as well as for all subsequent drivers (as shown in equations (A.17) and (A.20)), are proportional with $\mu$. We thus expect drivers with a higher $\mu$ to attach a higher value to the technology, and to be the ones equipped with incomplete penetration. Absent pricing, this should not affect the queuing pattern for a given $N_{e}$ and given $t_{q}$. But, it does suggest that the negative externality among equipped drivers becomes larger (the shift of the peak is more damaging, given their higher $\gamma$ ), while the positive externality on the unequipped drives becomes smaller (given their lower $\beta$ ). This in turn suggests that perfect competition may become relatively less efficient as the chances of overconsumption become bigger, while monopolistic supply may become relatively more efficient, essentially for the same reason. However, this reasoning takes $N_{e}$ and $t_{q}$ both as given; this of course is not correct and can only be remedied in a full equilibrium model with heterogeneity. We therefore once more emphasize the speculative nature of these predictions.

For ratio externality, the consequences are even harder to predict. This type of heterogeneity would produce a convex equilibrium travel delay function, with travelers arriving at the moment where its 
(absolute) slope equals the ratio of their value schedule delay to the value of time. Even given $N_{e}$ and $t_{q}$, we can therefore no longer use the equations from the main model to describe a traveler's willingness to pay for the technology. One may expect that the incentive from the technology to postpone the trip brings relatively great benefits to drivers with a relatively high $\alpha$ : the avoidance of queuing brings a relatively high benefit. Still, these drivers were driving - in the initial equilibrium - closer to the shoulders of the peak, anyway, so the question is whether this truly brings differentiated impacts such that high $\alpha$ drivers would be the first to adopt. It is probably safest, here, to make no prediction at all.

As far as the result on first-best pricing is concerned, the underlying principle that marginal cost pricing (under perfect competition) becomes optimal once all distortions are corrected for, appears so strong that it is likely to survive the introduction of heterogeneity in the model. 